\title{
Materias primas y objetos de prestigio en ajuares funerarios como testimonios de redes de intercambio en el Horizonte campaniforme
}

\author{
Corina LIESAU \\ Concepcion BLASCO \\ Departamento de Prehistoria y Arqueología - Universidad Autónoma de Madrid
}

\section{Resumen}

El trabajo da a conocer los resultados de recientes análisis realizados a diferentes componentes de los ajuares de enterramientos calcolíticos, especialmente campaniformes, de la región de Madrid. Con esta información, a parte de la clásica panoplia donada en los registros funerarios, otras entregas menos estudiadas evidencian con mayor rotundidad la capacidad adquisitiva y de control que determinados personajes pudieron tener sobre algunos recursos. Además del cobre y el oro, sobre los que ya se ha llamado la atención, el marfil y el cinabrio se perfilan como materias especialmente recurrentes en los registros funerarios campaniformes ricos. Su reiterada presencia indica que, aparte de controlar sus vías de distribución, se amplía el valor simbólico y de prestigio de los ajuares y los ritos de una élite cuyo poder adquiere aún más fuerza de la que se hasta ahora se le había asignado a partir de su clásica panoplia funeraria asociada.

Palabras clave: Calcolítico, Campaniforme, Valle Tajo, Ritual Funerario

\section{Summary}

This paper refers the results of recent analyses realized to different components of the chalcolithic grave goods, especially Bell Beaker ones in the Madrid region. New information about the materials of funerary records are not only an expression of the acquisitive capacity that certain prominent individuals could have by the control of some resources, not only copper and gold, but also ivory and cinnabar and their routes of distribution. In addition, the results reveal that the symbolic and prestige value of ivory and cinnabar is extended by an elite which power acquires more relevance which till now there had been assigned from the classic funerary package.

Keywords: Chalcolithic, Bell Beaker, Tagus valley, funerary ritual.

Se ha dicho reiteradamente que los enterramientos con cerámica campaniforme suelen poseer ajuares normalizados pero pocas veces se ha llamado la atención sobre la inclusión de determinadas materias primas, a veces de procedencia lejana o sobre el hecho de que los citados objetos

1 El trabajo ha sido financiado por los proyectos: Las sociedades calcolíticas y su marco temporal. Una revisión a la luz de nuevos datos (HAR2011-28731) Ministerio de Ciencia y Tecnología. I.P. Corina Liesau; y Patrimonio Arqueológico y y materias primas son patrimonio exclusivo de estas élites campaniformes, porque raras veces se estudian estos conjuntos asociados a enterramientos coetáneos que pueden coexistir en un mismo yacimiento en los que tales elementos y materiales están ausentes o son muy excepcionales. De

Documental de la Comunidad de Madrid (S2007/HUM 0543) Dirección General de Investigación de la Comunidad de Madrid. IP Concepción Blasco. 
hecho, es frecuente que los enterramientos calcolíticos sin campaniforme, practicados en fosas simples no tengan ajuar o éste se reduzca a donaciones de objetos cotidianos pertenecientes a piezas ya amortizadas. Generalmente se trata de enseres elaborados con materias primas procedentes de áreas de captación más o menos próximas: núcleos, lascas o láminas de sílex talladas con una tecnología sencilla, recipientes cerámicos y molinos de granito. Mientras que los restos cerámicos y la lítica pueden variar en calidad y cantidad, los molinos de granito suelen ser piezas habituales, hallándose en las tumbas colectivas varias piezas fracturadas y amortizadas (Liesau et al.; 2008, 109; Blasco et al., 2009, 45).

Menos frecuentes son los elementos de adorno, cuyos componentes siguen una arraigada tradición neolítica que utiliza en su elaboración piedras duras como la caliza, la variscita y otras piedras verdes. En una de las inhumaciones colectivas de Camino de las Yeseras se recuperaron diez cuentas discoidales de caliza de tamaño insignificante, pero de buena ejecución (5 $\mathrm{mm}$ de diámetro y 0.1 gr. de peso; Blasco et al., 2009, 49; Ríos y Liesau, 2011, 358). Sus reducidas dimensiones contrastan con algunas piezas decorativas de otras áreas peninsulares aunque son habituales en otras muchas regiones formando parte de adornos con diferentes composiciones y diversidad de materias primas (Pérez Arrondo y López de Calle, 1986:123).

En el valle medio del Tajo también las piedras de color verde empiezan a cobrar importancia en los ajuares funerarios calcolíticos de enterramientos sin campaniforme, se trata de materias primas de prolongada explotación desde el Neolítico, responden a dos variedades: la variscita (fosfatos de aluminio), poco habitual en el panorama geológico y las metavariscitas (silicatos de aluminio) que se encuentran en muchas más afloraciones, siendo las más próximas a nuestra área de estudio, distintas vetas del Sistema Central (Ríos, 2011, 515). Parece probado que los artesanos componían collares u otros adornos con cuentas de distinta naturaleza y procedencia, pues el efecto estético entre variscitas y metavariscitas, como sus cualidades para trabajarlas no representaban diferencias notables y todo parece indicar que, aunque este material es más habitual entre los grupos más tradicionales, sin campaniforme, también algunos de los que poseen esta cerámica, quizás mujeres y niños vinculados a las jefaturas, los seguían exhibiendo en sus ajuares.

Concretamente estas piedras las hemos documentado en uno de los enterramientos del yacimiento de Humanejos (Parla, Madrid) perteneciente a un individuo joven, posiblemente femenino, con importante ajuar que incluye, además de varios recipientes lisos y modestos punzones de cobre, un puñal también de este metal, de morfología foliácea muy similar a las alabardas de sílex y que además se ornamenta con un collar de cuentas verdes cuya analítica indica que se trata de silicatos de aluminio (Ríos y Liesau, 2011, 360, tabla 2 y 362) por tanto estos resultados confirman que se trata de metavariscitas. En Camino de las Yeseras, aunque no en contexto funerario primario, han sido recuperadas algunas piezas dispersas en el área central. Una de estas cuentas y una plaquita, ambas de variscita, se hallaron próximas a restos humanos dispersos, posiblemente expuestos en superficie por estar mordisqueados por cánidos, mientras que otras dos, de metavariscita, están relacionadas con otras actividades artesanales (Ríos, 2011; Ríos y Liesau, 2011, 358-364).

En la misma región de la cuenca media del Tajo, ya en la provincia de Toledo, se conocen otras cuentas de piedras verdes en dos tumbas diferentes de la necrópolis del Valle de las Higueras en Huecas (Bueno et al., 2005, 74 fig.5), en la Cueva 1 son de pequeño tamaño y forman parte de un collar en el que se combinan con ámbar que excepcionalmente pertenecen a un contexto con campaniforme; en la cueva 3 , una de las mujeres inhumadas ostenta, entre sus adornos personales, cuentas de collar de variscita (Bueno et al., 2005, 75). En este mismo yacimiento se han recuperado otras materias como el hueso o la concha, de larga tradición neolítica, o el ámbar material novedoso, que de momento no se ha encontrado en el registro de los yacimientos madrileños recientemente documentados.

En general, la austeridad de ajuares imperecederos en estas tumbas colectivas e individuales no campaniformes podría estar compensada con otros elementos de origen orgánico como la madera, vegetales comestibles, flores, aunque hasta la fecha hay pocos estudios en torno a estos vestigios, tenemos indicios de ello en dos tumbas colectivas de Camino de las Yeseras donde se han localizado de forma representativa polen de cere- 
al, restos de espigas y semillas de trigo desnudo (López Sáez, 2011, 256; Peña-Chocarro et al., 2011, 268-270; Cabanes, et al. 2011, 281-284). Los restos de cereal y la presencia de dos grandes piedras de molino en un lugar central de la base de ambas fosas funerarias no deja al azar la importancia de la actividad de la molienda como una tarea subsistencial en el registro funerario.

Las ofrendas de tipo animal son excepcionales y resulta difícil reconocer un patrón, tanto por falta de estudios, como por las dificultades de diferenciar entre restos depositados y material intrusivo. Son contados los casos de porciones más o menos completas (Liesau, 2011, 186), y aún más escasos los depósitos de animales completos como se han descrito en otros ámbitos peninsulares (Fabián, 2006, 315 Márquez, 2001 y Márquez y Jiménez, 2010, 219-315; Cámara et al., 2008; Lomba et al. 2009,153). Sin embargo, como se puede comprobar en diferentes yacimientos calcolíticos, caso de la Loma de Chiclana, El Espinillo, Las Matillas o Camino de las Yeseras una serie de animales o porciones relevantes de los mismos constituyen depósitos integrados en el espacio habitacional, cuidadosamente colocados en fosas o tramos de foso, por lo que el mundo animal parece cumplir con otras funciones que las de acompañar o alimentar al difunto u otras entes en el más allá (Díaz-Andreu et al., 1992,88 ; Baquedano et al.2000,26; Díazdel-Río, 2001, 201, Liesau et al. 2008, 106-108; Liesau, 2011; Daza, 2011).

Aunque no queremos extendernos en los estudios antropológicos que merecen un discurso más amplio, tan sólo enfatizar que durante el calcolítico no campaniforme predominan las inhumaciones colectivas con individuos de categoría de edades en los que predominan los infantiles y juveniles, mientras que en las inhumaciones individuales o dobles suele tratarse de personas adultas. En algunos casos, la forma de colocación de los cuerpos parece obedecer a un patrón, por el que se introducen primero los pies del difunto para dejar caer al azar el resto del cuerpo que a menudo adopta posturas forzadas y descuidadas (Gómez Pérez et al., 2011, 118-121).

A partir de mediados del III milenio estas prácticas conviven con las que establecen los grupos campaniformes cuyos enterramientos son más ricos en todos los aspectos funerarios, no sólo en lo que atañe a la estructura tumbal que abarca desde fosas simples a fosas con nicho, covachas e hipogeos abiertos en grandes espacios semiexcavados que delimitan un área funeraria de uso restringido (Blasco et al, 2005; Liesau et al., 2008, 111-115; Blasco, 2009; Ríos, 2011, 463-487). Las estructuras funerarias negativas de las cuencas sedimentarias madrileñas adaptadas a las condiciones geológicas del terreno conviven en la propia cuenca del Tajo con cuevas artificiales abiertas en ladera que se cierran con un muro de mampostería (Bueno, et al., 2005, 74; Bueno et al, 2007-08). La inversión de fuerza de trabajo no sólo es considerable en el vaciado de las estructuras negativas o en las cuevas abiertas en ladera, sino también en su cierre y cubrición de las mismas mediante mampuestos o grandes lajas y túmulos de cantos (Liesau et al., 2008, 111; Blasco et al., 2009, 54; Vega et al., 2010; Ríos, 2011).

Además, los grupos campaniformes imponen para sus integrantes unos rituales mucho más normalizados que incluyen siempre la donación de recipientes, generalmente ornamentados, hechos específicamente para su amortización en ceremonias fúnebres a los que se suman, de manera puntual, en el caso de los enterramientos de individuos destacados, determinados ornatos y donaciones "singulares" como objetos de metal, especialmente armas, y adornos realizados con materias primas exóticas.

En efecto, desde hace ya tiempo la amortización de objetos singulares en los enterramientos campaniformes ha llamado la atención de los investigadores quienes han repetido la idea de la existencia de una normativa relativamente repetitiva que incluye un "set" de recipientes cerámicos -vaso cuenco y cazuela-, la panoplia formada por una o más puntas palmela y puñal de lengüeta $y$, con relativa frecuencia: brazal (afilador para algunos autores) y elementos de ornato personal entre los que destacan, por su frecuencia, los botones de perforación en "V" (Delibes, 1977, 116-118; Harrison, 1977, 39-42; Uscatescu, 1992; Garrido, 2000, 323-325). Si bien pocas veces se da la combinación completa y pueden producirse múltiples combinaciones que, en ocasiones, incluyen también otros objetos de prestigio como algunas joyas de oro, adornos realizados con materias exóticas como el marfil, además de cinabrio aplicado sobre el cuerpo y objetos, o simplemente presente en el lecho preparatorio. Se trata 
de piezas con elevado coste de elaboración, tanto por la procedencia lejana de la mayoría de las materias primas como por el empleo en su fabricación de técnicas especializadas. Teniendo en cuenta distintos factores que singularizan estos ajuares podemos clasificarlos en los siguientes grupos:

1- Objetos hechos con materiales locales pero tipológica-estética y posiblemente también simbólicamente- reservados a los ajuares funerarios como son las cerámicas campaniformes.

2- Útiles fabricados con "nuevos materiales" que requieren también técnicas nuevas, es el caso de la metalurgia y, especialmente, las armas, la metalurgia más compleja del momento.

3- Piezas realizadas también con "nuevos materiales" que, además son exóticos, procedentes de intercambios a larga distancia: marfil y oro.

\section{4- Empleo del cinabrio en el ritual funerario.}

De estos cuatro grupos nos interesa destacar los dos últimos porque son sobre el que se ha hecho menos hincapié y, pocas veces se ha tratado de manera conjunta, es decir teniendo en consideración el conjunto de materias primas que conviven en un mismo ajuar, porque no sólo son de carácter exótico por su lejana procedencia, sino que además pertenecen a elementos con una escasa oferta geológica o biológica: marfil, oro y cinabrio. A la luz de los nuevos hallazgos son tres materias primas especialmente recurrentes y exclusivas de las élites cuya procedencia distinta amplía nuestra perspectiva y obliga a valorar diferentes circuitos, si bien hay que reconocer que cinabrio y oro se concentran en distintos puntos del occidente peninsular (Figura 1).

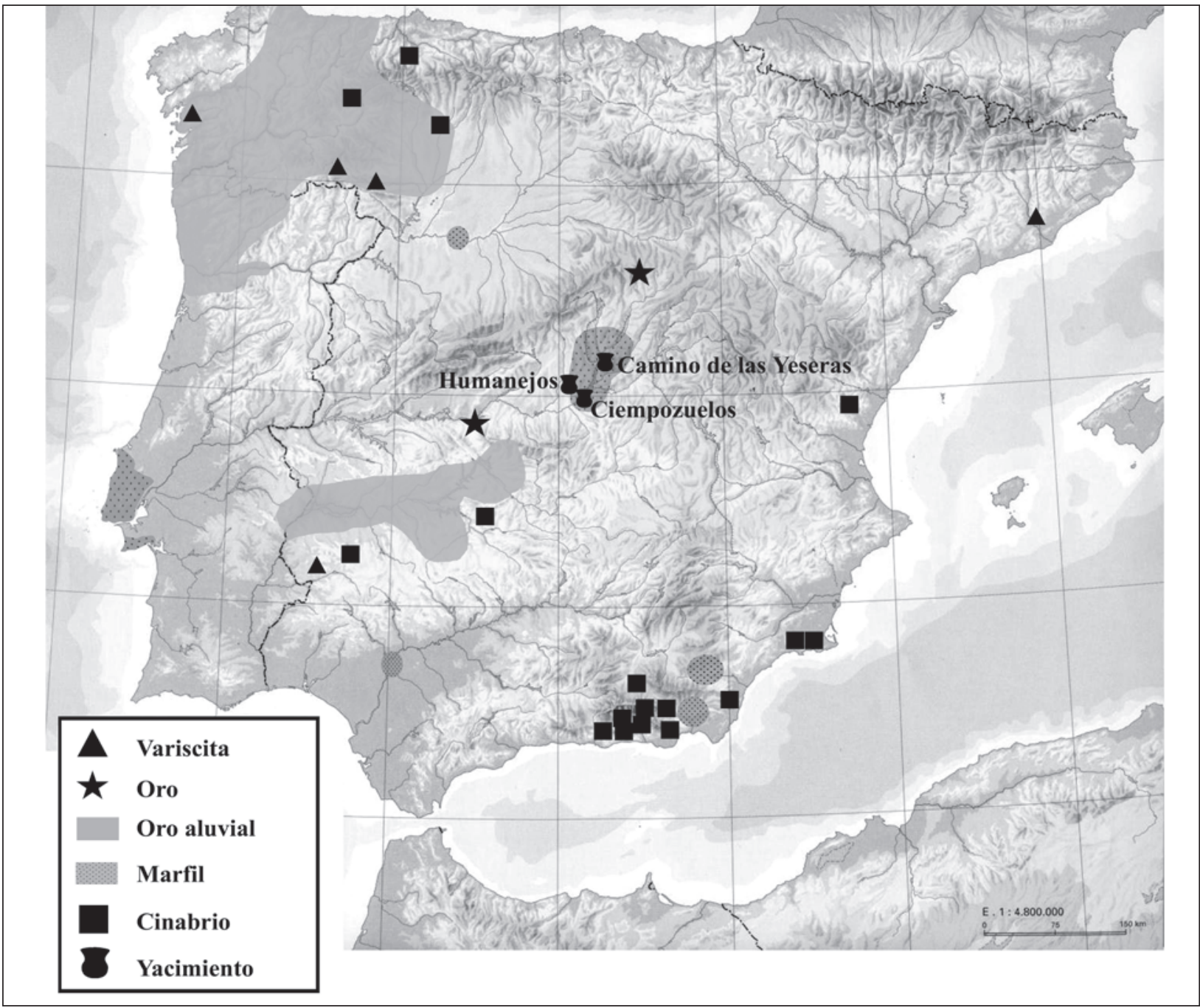

Figura 1. Distribución de las principales fuentes de abastecimiento de las materias primas estudiadas: oro (a partir de A. Hernando, (1983) y Montero y Rovira (1991), cinabrio, a partir de Delibes (2000); Hunt y Hurtado (2010) y marfil, a partir de Schuhmacher et al. (2009); Liesau y Moreno (2011). 
Un ejemplo claro lo ha proporcionado la analítica aplicada a los ajuares campaniformes de marfil en Camino de las Yeseras y otros contextos campaniformes cercanos, todos ellos pertenecientes a la provincia de Madrid. Incluso analíticas de piezas de excavaciones antiguas han aportado datos de gran interés revelando que son, probablemente, bastantes las cuentas, botones y otros adornos que han pasado desapercibidos en su calidad de marfil, por lo que la presencia de esta materia prima es mucho más frecuente de los que hasta la fecha se había pensado. Así lo demuestra la recuperación de que tres botones custodiados en la RAH, posiblemente procedentes de alguno de los famosos enterramientos hallados en la necrópolis Ciempozuelos (Riaño et al. 1894; Blasco et al., 1998), recopilados por Garrido (2000: lám. 28) e inicialmente identificados como de hueso, aunque en una posterior catalogación se alude a la posibilidad de que se tratase de hueso o de marfil (Almagro et al., 2004, 97) y que en fechas próximas podremos confirmar definitivamente, al estar pendientes de obtener los resultados de la espectrometría de infrarrojos transformada por Fourier (FTIR). A nuestro entender, el estudio macroscópico -patrón de fracturación laminar característico de las bandas de dentina de proboscídeos- apunta a la naturaleza ebúrnea de todas ellas, y presentan además adherencias de cinabrio como veremos más adelante.

En Camino de las Yeseras se ha exhumado una tumba individual campaniforme que destaca por su singular ajuar, pertenece a un joven adulto que posee un tocado áureo, una cazuela campaniforme con grabados de cérvidos, además de tres botones de perforación en "V" y una escasa treintena de cuentas de collar realizadas en marfil (Liesau et al, 2008,113-116; Blasco et al., 2009, 59-61;) (Figura 2, 1-5). A pesar de que el esqueleto, como la mayoría de las piezas ebúrneas presenta un deficiente estado de conservación, ha sido posible obtener espectroscopías (FTIR) de una decena de estas piezas, como también microtomografías computerizadas $(\mu-\mathrm{CT})$ realizadas por el Dr. Banerjee en la Universidad de Maguncia. Los resultados han sido interesantes al revelar un empleo mayoritario de marfil de elefante antiguo (Elephas (Paleoloxodon) antiquus), mientras que una pequeña esquirla de marfil tomada como muestra de control ha revelado un perfil característico del elefante africano de la estepa (Loxodonta africana) (Liesau et al., 2011, 382; Liesau y Moreno, 2011).

En el yacimiento de Humanejos también se ha localizado un ajuar selecto campaniforme con un destacado lote de piezas: botones de perforación en "V" hemiesféricos y de tortuga, así como cuentas de collar con morfología de tendencia bicónica muy similares a las del enterramiento de Camino de las Yeseras que acabamos de mencionar (Figura 2, 7-11). También pendiente de obtener los resultados en relación con su determinación taxonómica, su naturaleza ebúrnea parece estar fuera de toda duda (Ríos y Liesau, 2011, 365-367).

Todos estos nuevos hallazgos y la revisión de otros tantos antiguos no hace más que rellenar un gran vacío geográfico que hasta la fecha se ha tenido de inventarios ebúrneos madrileños, suscitando nuevos retos de investigación ante el origen de esta materia prima tradicionalmente considerada como procedencia extrapeninsular y como producto de prestigio en intercambios a larga distancia, es decir, entre el Norte de África y la Península ibérica (Harrison, 1977: 39-43; Harrison y Gilman, 1977).

El empleo mayoritario de un marfil de elefante extinguido durante el Pleistoceno Superior puede indicarnos la explotación de unos recursos en condiciones favorables de conservación más o menos próximos. Aunque sin descartar una producción local o cercana a partir de la de colmillos de elefantes extinguidos recuperados de las terrazas fluviales de las cuencas madrileñas (Figura 1), nada impide suponer que también circulasen partidas en otros ámbitos peninsulares o extrapeninsulares junto a los marfiles de elefantes existentes en paisajes africanos. De hecho, la variedad taxonómica de circulación de marfiles es amplia desde el inicio del Calcolítico para el Sur y Oeste peninsular, y a los que tenemos que añadir marfil del elefante asiático, y de cachalote como apuntan Schuhmacher y otros (Schumacher y Cardoso, 2007; Schuhmacher et al., 2009). Durante la Edad del Bronce continúa habiendo registros que muestran también el empleo de marfil de elefante antiguo en el ámbito argárico, aspecto que no hace más que enfatizar la explotación o 


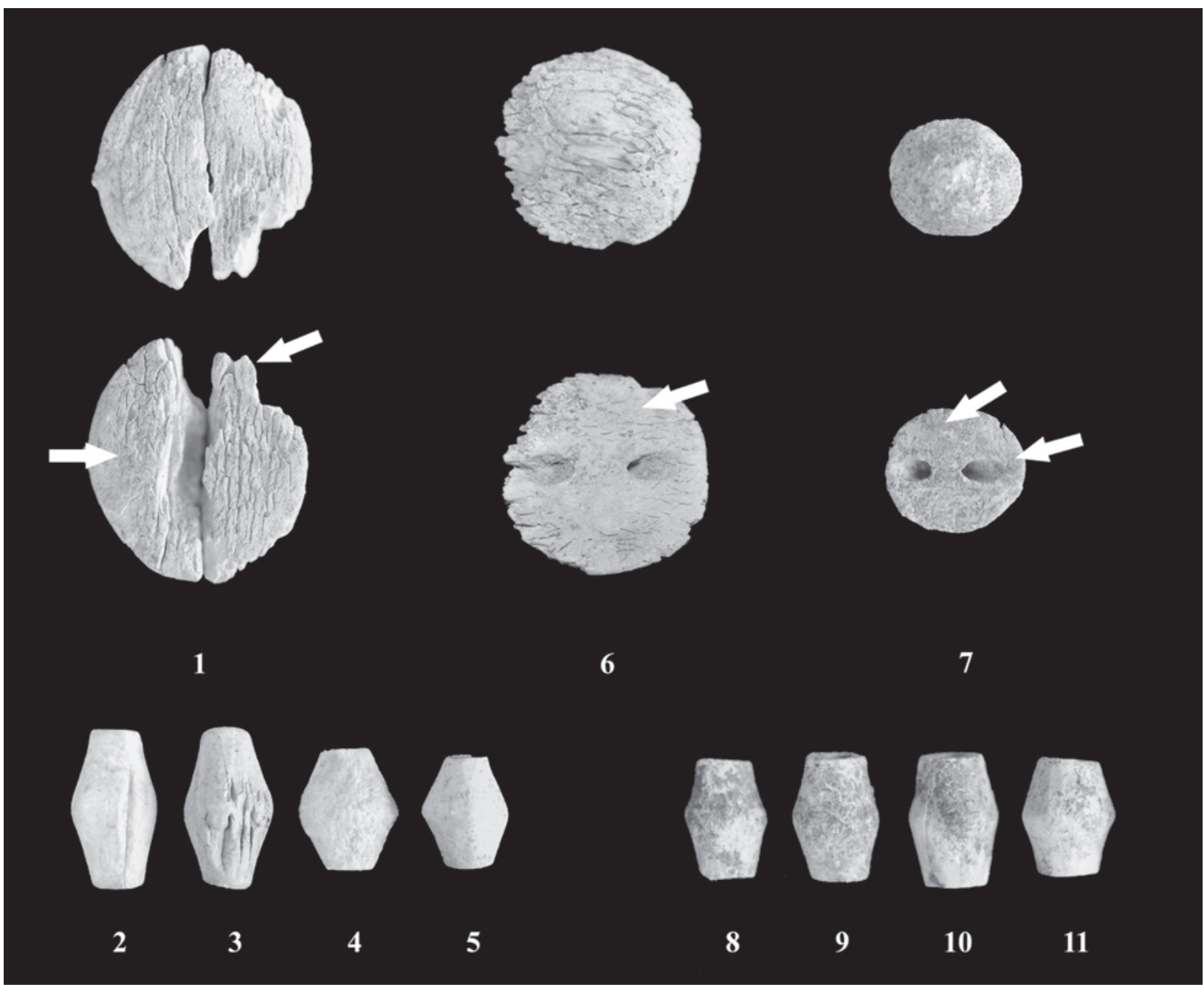

Figura 2. Selección de piezas ebúrneas de Camino de las Yeseras: nº 1-5; nº 6: Cuesta de la Reina, (Almagro et al., 2004; $\mathrm{n}^{\circ}$ inv. 1640/2b); n ${ }^{\circ}$ 7-10 de Humanejos (Ríos y Liesau, 2011: 366).

Las flechas blancas indican restos visibles de cinabrio adherido a la superficie.

intercambio de una amplia gama de materiales ebúrneos a lo largo del tiempo (Liesau y Schuhmacher, 2011).

Podemos concluir este apartado en, que pendientes de futuros estudios en torno al origen local o foráneo de este selecto material, los hallazgos en diferentes yacimientos madrileños se reducen puntualmente a determinadas inhumaciones campaniformes cuya riqueza en ajuares cerámicos y/o metálicos refleja el prestigio y exclusividad de sus portadores. No parece tampoco casualidad de que se trate fundamentalmente de individuos masculinos. Aunque con ciertas reservas para el yacimiento de la Cuesta de la Reina, no descartamos también en este caso el que el ajuar más destacado pudiera corresponder a una inhumación secundaria de un varón maduro trepanado (Sampedro y Liesau, 1998, 45-49; Liesau y Pastor, 2003). Esta asociación estaría en consonancia con los ajuares de destacados varones maduros (contemporáneos argáricos, donde botones de diversas tipologías presentan un valor añadido al ajuar metálico (López Padilla, 2006 a y b).

Los nuevos hallazgos han ampliado también sensiblemente el número de contextos calcolíticos campaniformes con elementos de oro, y se ha demostrado que, posiblemente en excavaciones poco cuidadas, no se haya recuperado la totalidad de este material pues a las diademas de cintas conocidas hasta ahora, tenemos que sumar la existencia de ornatos compuestos por pequeñas cuentas que están presentes en un número muy alto de tumbas, certificando que la metalurgia del oro debió de ser bastante más habitual de lo que se había venido pensando (Blasco et al., 2009, 60; Blasco y Ríos, 2010). 
Con respecto al oro, los análisis han proporcionado una extraordinaria homogeneidad entre todos los elemenos recuperados ya que contienen entre el 91 y el $95 \%$ de oro y tasas de plata de entre el 7 y el $5 \%$ a excepción de una placa que es de oro prácticamente puro: 99'9\% y que está realizada sobre una lámina sensiblemente más gruesa que el resto de las piezas (Placa superior de la figura 3.2). Dicha homogeneidad ha sido contrastada satisfactoriamente tras el análisis de tres cuentas tubulares por el método PIXE en el acelerador AGLAE del Museo del Louvre (por la Dra. María Filomena Guerra, a quien agradecemos su colaboración). En dichos análisis se detectaron también cantidades de cobre del orden de 0,02 a $0,05 \%$. De estos datos se desprende algo habitual en la orfebrería calcolítica peninsular: la procedencia aluvial del oro en el que se confeccionaron estas joyas, caracterizada por una escasa presencia de cobre, no superior al 0'1\% (Perea
1991, 31-35), una evidencia que confimaron los análisis realizados por Hartmann (1982) quien los englobó dentro del que denominó grupo $\mathrm{S}$.

Están por hacer los estudios analíticos de las distintas cuencas auríferas (Montero y Rovira 1991) y no es menos cierto que en la provincia de Guadalajara, lindando con la de Madrid, han estado operando lavaderos auríferos hasta mediados del siglo pasado en La Nava de Jadraque y Arroyo de la Fragua y también, más cerca, en Palancares, en la región del alto Jarama. Pese a ello no descartamos que estas joyas, lo mismo que la diadema procedente del Dolmen de Entretérminos o la lámina en espiral del Vertedero de Salmedina (Berzosa y Flores 2005, 483), llegaran a su destino ya confeccionadas, procedentes de posibles talleres especializados, seguramente instalados en el NO peninsular donde los hallazgos áureos de piezas con características y tipologías similares

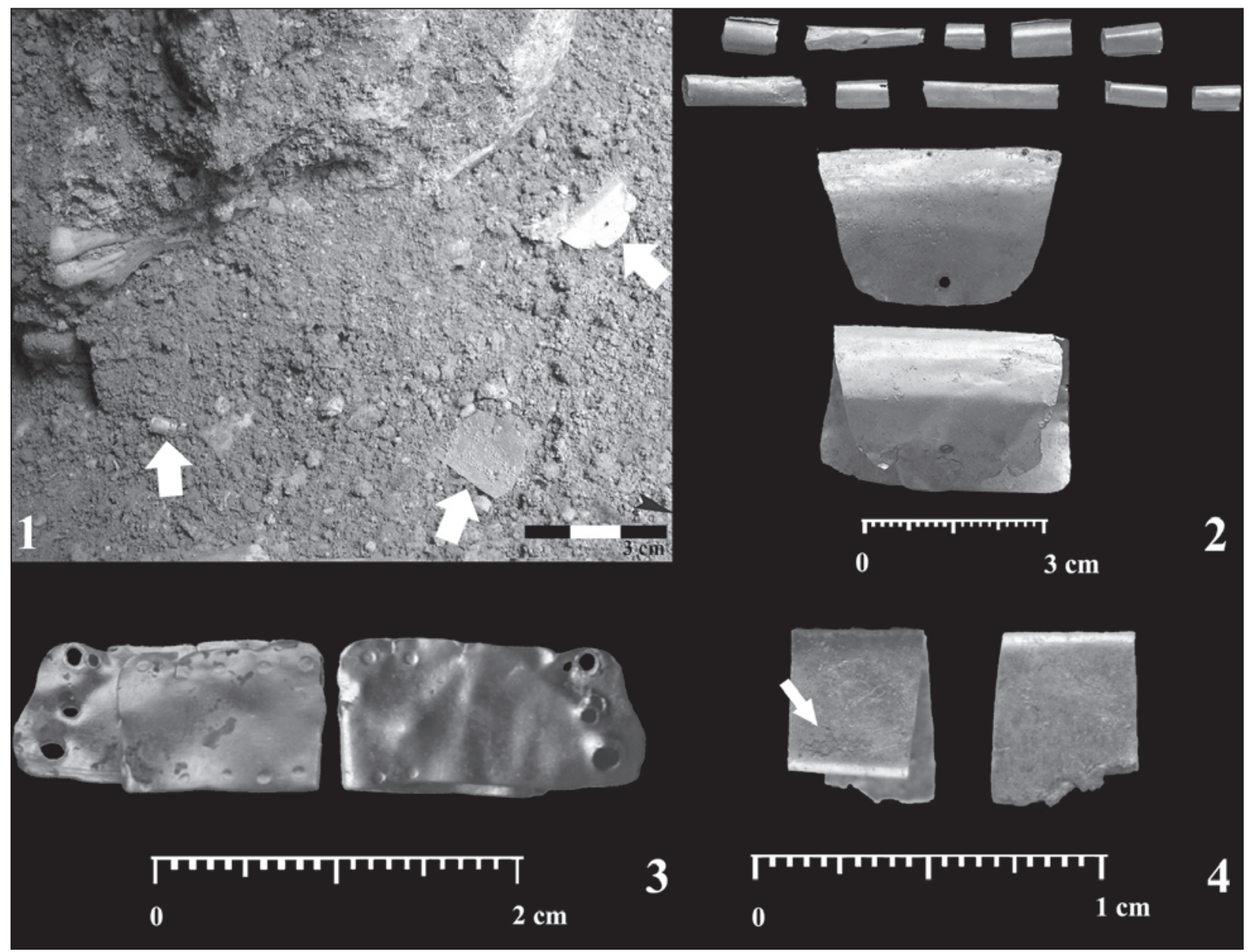

Figura 3. Camino de las Yeseras: 1.- detalle del individuo del hipogeo del área funeraria 2 con parte de los elementos áureos que componen la diadema. 2- Selección de piezas de oro de la diadema. 3.- Laminita de oro repujado recuperada entre las piedras de relleno del hipogeo del área funeraria 1. Humanejos: 4. Laminita de oro con restos de cinabrio adherido del hipogeo UE 455. 
son especialmente frecuentes y desde donde se pudieron distribuir a todo el territorio peninsular (Figura 1).

No menos interesante es la asociación del oro y el marfil con el uso del cinabrio o el bermellón un "sulfuro de mercurio reducido a polvo y lavado" de color mucho más encendido que el ocre (Delibes, 2000, 228). Mineral empleado desde tiempos antiguos tanto de forma aislada o combinación con el ocre, se conoce con mayor frecuencia en diferentes contextos funerarios neolíticos y habitacionales (Delibes, 1995, 2000; Domínguez y Morata, 1995; García Borja et al., 2006). Mientras que las variedades de ocres son muy abundantes en la naturaleza a nivel mundial, el cinabrio es un recurso mineral poco frecuente, presente en algunas vetas asociadas a actividades volcánicas, a piritas, marcasitas, cuarcitas, calcitas y yesos (Eastaugh et al., 2005, 105).

Los análisis mineralógicos y químicos han permitido diferenciar, tanto muestras de pigmentos rojos recuperados de sedimentos y piezas de excavaciones actuales -Camino de las Yeseras, Humanejos, La Magdalena-, como de otras antiguas -Cuesta de la Reina, (Ciempozuelos)-. Los resultados han sido concluyentes en el empleo de cinabrio en tumbas campaniformes con ricos ajuares para Camino de las Yeseras, Ciempozuelos y la Magdalena, mientras que en Humanejos también se hace extensivo a algunas de las inhumaciones más singulares contemporáneas al campaniforme pero sin esta cerámica (Ríos y Liesau 2011, 367-368).

Desde el punto de vista cronocultural el empleo del cinabrio, mezclado con ocre se remonta a una etapa precampaniforme; concretamente en la zona central de Camino de las Yeseras ha sido posible documentar un pequeño aglomerado en estado puro y un fragmento cerámico de excelente acabado con una decoración, posiblemente simbólica, ejecutada con puntos impresos rellenos con ocre (Ríos y Liesau, 2011 361, tabla 3-5).

Pendientes de conocer análisis más específicos de isótopos de plomo para determinar el origen de las explotaciones del cinabrio durante el Calcolítico, parece que las características de las muestras de cinabrios madrileños procedentes de Humanejos tienen ciertas concordancias con los de las minas de Almadén (Hunt, com.verb.) y en este sentido también una serie de análisis de contextos funerarios calcolíticos apuntan hacia esta importantísima mina manchega, descartando otros posibles depósitos extremeños o andaluces (Hunt y Hurtado, 2010, 131).

El hecho cierto es que la detección y el análisis de composición química de los pigmentos rojos en las tumbas campaniformes es otro ítem más a tener en cuenta en la valoración de los elementos de prestigio, así como detectar si aparece de forma más o menos pura o estaba mezclado con tierras ocres. El hallazgo de un atesoramiento de "almagras" en un hoyo del yacimiento de Soto de Henares muestra la importancia de este tipo de registros (Galindo et al., 2009, 416) y llama la atención el escaso interés mostrado en muchas intervenciones arqueológicas durante estos últimos años, cuando parece formar parte de un gesto funerario bastante normalizado.

Tanto si el cinabrio se empleaba como tinte para las mortajas, como si hubo antes del cierre de la tumba actos de espolvorear zonas concretas del cuerpo como la cabeza, brazos, rodillas, pies y determinados ajuares, se trata en de evidencias bien documentadas en Camino de las Yeseras, Humanejos, Salmedina (Ríos y Liesau, 2011, 361-362, tabla 3, 6 a 10; Berzosa y Flores, 2005, 559), como también en otras necrópolis de la Cuenca del Tajo, caso del valle de Las Higueras (Bueno et al., 2005). En el ámbito madrileño se dispone además de un claro precedente del horizonte campaniforme, de vestimentas coloreadas de ocres (no cinabrio) en la cueva de Juan Barbero (Rovira y Sanz, 1984, 101-102).

La tinción de la parte basal de botones con perforación en $\mathrm{V}$, hallados en tumbas campaniformes como Cuesta de la Reina, Camino de las Yeseras o en una tumba de Humanejos nos acerca una vez más al ámbito argárico donde este tipo de fenómeno se ha relacionado con piezas teñidas de cinabrio al estar cosidas sobre prendas impregnadas con esta sustancia (Delibes, 2000, 227; López Padilla, 2006b, 29). Pero también otra evidencia nos acerca a otra práctica argárica bien documentada, se trata de un individuo campaniforme hallado en Humanejos, donde se puede observar con nitidez dos franjas rojas paralelas entre sí que rodean frente y parietal y cuyo análisis ha dado 


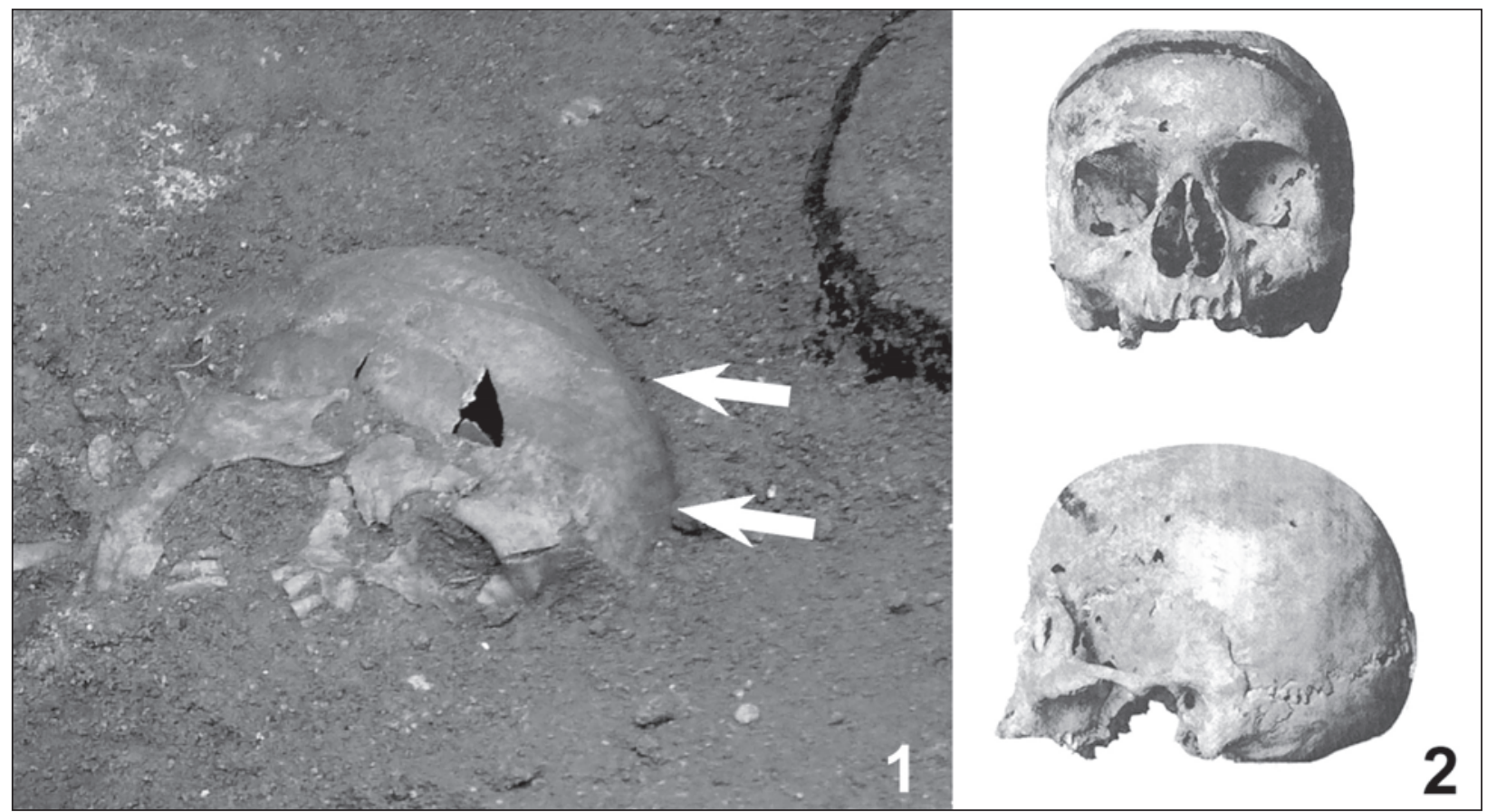

Figura 4 1.- Detalle del cráneo de un enterramiento campaniforme de Humanejos con impresión a banda doble de cinabrio. (Fotografía, Raúl Flores). 2.- Cráneo de El Argar (tumba 356), mostrando la faja de cinabrio sobre frente y oreja, según H. y L. Siret (1890, lám. XX, 1 y 2).

como resultado ser cinabrio (Figura 4.1). Este tipo de huella ha sido extensamente discutida por los hermanos Siret (1890: 195-202) en diferentes inhumaciones argáricas, como también por Delibes $(2000,226)$. La interpretación más plausible es que se trata de cintas embadurnadas en rojo bermellón que con el tiempo desaparecieron, dejando una banda en rojo sobre frente, parietales y occipital, por lo que se descartan actos de mero embadurnamiento de los huesos descarnados previamente (Figura 4.2).

Todo ello nos lleva a considerar, no sólo que su presencia no es aleatoria sino que también forma parte de una intencionalidad en distinguir a estos personajes con una donación que, al igual que el oro, y el marfil es de alto coste de adquisición y, por tanto, al alcance de unos pocos. Más allá de su calidad estética, la visión de cuerpos con vestimentas rojas o espolvoreados con cinabrio y complementados con oro y marfil darían un efecto muy destacado por su coloración y espectacular brillo durante la ceremonia funeraria. No obstante, el empleo del cinabrio podría estar también encaminado a favorecer la conservación de los cuerpos, contribuyendo a su momificación o, al menos a la desaceleración del proceso de descomposición (Delibes, 2000, 230). Consideramos que para determinadas construcciones funerarias como los grandes hipogeos puede tratarse de una práctica muy necesaria, teniendo en cuenta que su vaciado requiere de una importante inversión en fuerza trabajo debido a su profundidad, complejidad y aparatoso cierre por lo que el tiempo empleado en estos trabajos requiere una conservación de los cadáveres en las mejores condiciones posibles antes de su definitiva inhumación. Camino de las Yeseras es un buen ejemplo en el que, al menos, en dos áreas funerarias, los hipogeos allí documentados sorprenden por su monumentalidad y cierre, al igual que otra en Humanejos (Flores, 2011, 13,14).

\section{Algunas reflexiones finales sobre RITUALES FUNERARIOS CAMPANIFORMES}

Cabe destacar que mientras las materias primas y objetos privativos de los enterramientos singulares campaniformes son una novedad que indica una interacción que incorpora la apertura a nuevos circuitos, los enterramientos destacados no campaniformes incluyen objetos de adorno confeccionados en piedras verdes (variscitas y metavariscistas y objetos de cobre que se reducen a punzones u otros útiles cotidianos. Cobre y pie- 
dras verdes pueden proceder, en muchos casos de las mismas afloraciones por lo que su obtención se canalizaría a través de los mismos circuitos. Ello explica que durante el Calcolítico las procedencias de variscitas y metavariscitas sean de áreas geográficas mucho más variadas que en el Neolítico

Sin duda, para los registros funerarios campaniformes el significado simbólico del oro, así como del resto de los elementos y materias primas de prestigio se mantuvo más allá de su amortización como ajuar y fue posiblemente la causa de las reiteradas manipulaciones que sufren los conjuntos tumbales más relevantes. Seguramente no se buscaba solo el oro o alguna de las otras materias exóticas que pudieran contener por la codicia de obtener un beneficio material, sino por la apropiación de lo excepcional, por su significado, tratando de emular, mediante la posesión de las enseñas simbólicas, el status que representan. Quizás sea significativo de esta intención el hecho de que no se trata de una rapiña exhaustiva, sino que intencionadamente las cerámicas se rompen para sustraer sólo una parte de cada uno de los recipientes, de igual manera, se exhuman los restos óseos del difunto más representativos y se dejan pequeñas porciones, aspecto bien documentado en Camino de las Yeseras o también se traslada parte de un cuerpo al inhumar otro dentro de la misma tumba, caso de Humanejos y que demuestra sucesivas aperturas que se acometen en las tumbas colectivas campaniformes (Gómez, et al. 2011).

Con todo ello se podría plantear la hipótesis del permiso que se le concede al difunto de disfrutar durante un cierto tiempo de ajuares ligados a una estirpe o un núcleo familiar con principios hereditarios bien establecidos, y que una vez pasado un tiempo prudencial - descomposición del cadáver, inclusive los tendones y ligamentosse reabren las tumbas por sus seres más allegados para poder, no sólo llevarse consigo elementos de prestigio, sino también partes significativas del cuerpo, especialmente cráneos y huesos largos. Los cráneos cobran un especial significado cuando en Camino de las Yeseras se han recuperado restos craneales en numerosas estructuras domésticas, además en una "cabaña o recinto funerario" colmatando un pequeño túmulo funerario de una covacha, o también la colocación de huesos largos humanos acompañados por un cuenco y un vaso liso en una gran fosa campaniforme abierta en varias ocasiones y posteriormente cuidadosamente cerrada, incluyendo un sacrificio de canes (Blasco et al., 2005:463; Liesau et al., 2008, 116117, Blasco et al., 2009, 64-67).

El acto de sustracción o traslado de restos bien documentado en los yacimientos anteriores no es un fenómeno nuevo ni exclusivo de su necrópolis ya que está documentado en contextos funerarios campaniformes de otras zonas peninsulares como en Aldeagordillo (Ávila) (Fabián, 1992, 2006) y también queda patente en Almenara de Adaja (Valladolid) donde tan sólo dos costillas humanas se incorporan a un sedimento ceniciento con decenas de vasos, cazuelas y cuencos, uno de ellos con una decoración simbólica de ciervos y soles, huesos de animales, además de evidencias de restos de bebidas alcohólicas como la cerveza (Guerra y Delibes, 2004; 2005; Delibes y Herrán, 2007: 195). En la necrópolis francesa de Georges Besse la extracción sistemática del esplacnocráneo revela una retirada de esta parte esquelética un tiempo aproximado de dos años, una vez descompuesta las partes blandas y los ligamentos (Tchérémissinoff et al., 2011: 172-173).

Aunque todavía nos encontramos lejos de desvelar el significado real de estas profanaciones, resulta especialmente llamativo que los sujetos que llevaron a cabo los expolios se mostraran respetuosos y, una vez finalizada su acción, volvían a colocar rellenar las tumbas con sedimento y cubrirlos con un túmulo de piedras. Aunque no podemos descartar que se trate de actos que pretenden pasar desapercibidos y con la intención de volver al estado primario de la cobertura original de la tumba como argumentan Tchérémissinoff $e t$ al. (2011: 173) parece más probable que nos encontremos ante un acto ritual, consentido por toda o parte de la comunidad la cual participaba en él de manera directa o indirecta. A favor de esta segunda hipótesis, relacionada con un acto ritual, está la premeditada selección de algunas porciones representativas del esquelético y en la intencionada rotura de los contenedores cerámicos con el fin de mantener una parte del todo en el depósito funerario, o introducir nuevas piezas en las que no descartamos que fuesen acompañados por 
actos libacionales e inclusión parcial o total de recipientes cuyos contenidos, entre otros, demuestran evidencias de bebidas alcohólicas (Guerra, 2006; Rojo et al., 2006; Delibes y Herrán, 2007). Frente a ello, el oro y otros objetos de marcado significado simbólico eran sustraídos. Una vez terminada la acción los participantes o sus colaboradores procedían a la reconstrucción del paisaje funerario manteniendo la visualización jerárquica de las tumbas.

Por otra parte en el caso concreto de Camino de las Yeseras, la donación de materias lejanas y de alto coste cobra una especial relevancia pues, a diferencia de otros ajuares campaniformes que evocan el prestigio de los difuntos fundamentalmente por la presencia de armas o por la suma de armas y ornatos de adorno realizados con materias preciosas, la distinción social se manifiesta únicamente por la donación de estos ricos ornatos personales singulares. El clásico set campaniforme queda sustituido por la generosa donación de las tres materias exclusivas y costosas -oro, marfil y cinabrio- junto con tan sólo uno o dos recipientes cerámicos. En consecuencia, el prestigio y la función no viene determinada por las armas y vasos como recipientes de bebida, sino también por otros elementos de tanta o más significación para con ello distinguir a determinados personajes que rompen con los esquemas tradicionales de poder y prestigio basados en parámetros coercitivos.

\section{Agradecimientos}

Agradecemos a Raúl Flores, y a César M. Heras directores de las excavaciones de Humanejos (Parla), La Magdalena (Alcalá de Henares) por facilitarnos muestras y sedimentos del yacimiento para poder realizar diferentes análisis, como también a Martín Almagro Gorbea, el poder estudiar los botones de Cuesta de la Reina custodiados por la RAH.

\section{Bibliografía}

ALMAGRO-GORBEA, M.; CASADO, D.; FONTES, F.; MEDEROS, A. y TORRES, M. (2004): Prehistoria. Antigüedades Españolas I. Real Academia de la Historia. Catálogo del Gabinete de Antigüedades. Madrid.
BAQUEDANO, M I., BLANCO, J. F., ALONSO, P. y ÁLVAREZ, Ma D. (2000): El Espinillo: un yacimiento calcolítico y de la Edad del Bronce en las terrazas del Manzanares. Arqueología, Paleontología y Etnografía 8. Madrid.

BERZOSA, R. y FLORES, R. 2005: "El conjunto funerario campaniforme del Vertedero de La Salmedina (Distrito de Villa de Vallecas, Madrid", en Rojo et al., (coord.): El Campaniforme en la Península Ibérica y su contexto europeo, Universidad de Valladolid: 481494.

BLASCO BOSQUED, C.; BAENA PREYSLER, J. y LIESAU VON LETTOW-VORBECK, C.: La Prehistoria madrileña en el Gabinete de Antigüedades de la Real Academia de la Historia. Los yacimientos Cuesta de la Reina (Ciempozuelos) y Valdocarros (Arganda del Rey): Depto. de Prehistoria y Arqueología de la U.A.M. Patrimonio Arqueológico del Bajo Jarama,3.

BLASCO; C., LIESAU, C., DELIBES, G., BAQUEDANO, E. y RODRÍGUEZ CIFUENTES, M. (2005): "Enterramientos campaniformes en ambiente doméstico: el yacimiento de Camino de las Yeseras (San Fernando de Henares, Madrid)", en Rojo et al., (coord): El Campaniforme en la Península Ibérica y su contexto europeo, Universidad de Valladolid.

BLASCO C., DELIBES, G., BAENA, J., LIESAU, C. y RÍOS, P. (2007): "El poblado calcolítico de Camino de las Yeseras (San Fernando de Henares, Madrid). Un escenario favorable para el estudio de la incidencia campaniforme en el interior peninsular" Trabajos de Prehistoria, 64 (1): 151163.

BLASCO, C., LIESAU, C., RÍOS, P., BLANCO, J. F., ALIAGA, R., MORENO, E. y DAZA, A. (2009): "Kupferzeitliche Siedlungsbestattungen mit Glockenbecher- und Prestigebeigaben aus dem Grabenwerk von el Camino de las Yeseras (San Fernando de Henares, prov. Madrid). Untersuchungen zur Typologie des Grabritus und zu dessen sozialer Symbolik“. Madrider Mitteilungen, 52: 40 - 70 .

BLASCO; C., LIESAU, C., y RÍOS, P. (eds.) (2011): Yacimientos calcolíticos con campaniforme en la Región de Madrid: Nuevos estudios. Patrimonio Arqueológico de Madrid, 6.Universidad Autónoma de Madrid.

BLASCO, C. y RÍOS, P (2010): "La función del metal entre los grupos campaniformes. Oro versus cobre. El ejemplo de la Región de Madrid". Trabajos de Prehistoria, 67, $\mathrm{n}^{\circ}$ 2, 359-372. 
BUENO, P., BARROSO, R. y BALBÍN, R. (2005): "Ritual campaniforme, ritual colectivo: La necrópolis de cuevas artificiales del Valle de las Higueras, Huecas, Toledo". Trabajos de Prehistoria, 62, n 2, 67-90.

BUENO, P., BARROSO, R. y BALBÍN, R. (2007-08): "Campaniforme en las construcciones hipogeas del megalitismo reciente al interior de la Península Ibérica". Veleia, 24-25:771-790.

CABANES, D., ALIAGA, R. y MORENO, E. (2011): "Los fotolitos de silíceo". En Blasco et al. (eds.): 277-287.

CÁMARA, J. A. (2001): El ritual funerario en la Prehistoria Reciente en el Sur de la Península Ibérica. British Archeological Report, Interantional Series, 913. Oxford 457-479.

CÁMARA, J. A.; LIZCANO, R. PÉREZ, C. y GÓMEZ, E. (2008): “Apropiación, sacrificio, consumo y exhibición ritual de los animales en el Polideportivo de Martos. Sus Implicaciones en los orígenes de la desigualdad social". Cuadernos de Prehistoria de la Universidad de Granada, 18:55-90.

DAZA, A. (2011): "Los depósitos de perros". En Blasco et al. (eds.), 211-222.

DELIBES, G. (1977): El Vaso campaniforme en la meseta Norte española. Studia Archaeologica 46, Universidad de Valladolid.

DELIBES, G. (1995): "Ritos funerarios, demografía y estructura social entre las comunidades neolíticas de la submeseta Norte". En: Fábregas Valcarce et al (eds.):61-94.

DELIBES, G. (2000): Cinabrio, huesos pintados en rojo y tumbas de ocre: ¿prácticas de embalsamiento en la Prehistoria? En: Olcina, y Soler, J. (coord.): Scripta in Honorem Enrique A. Llobregat Conesa. Alicante: 223-235.

DELIBES, G. y HERRÁN, I. (2007): La Prehistoria. Biblioteca básica de Valladolid. Diputación de Valladolid. Valladolid.

DÍAZ ANDREU, M., LIESAU, C. y CASTAÑO, A. (1991): "El poblado calcolítico de La Loma de Chiclana (Vallecas, Madrid). Excavaciones de urgencia realizadas en 1987". Arqueología, Paleontología y Esnografía, 3, 31-116.

DÍAZ -DEL- RÍO, P. (2001): La formación del paisaje agrario: Madrid en el III y II milenio BC. Arqueología, Paleontología y Etnografía 7. Madrid.

DOMINGUEZ BELLA, S. Y MORATA CÉSPEDES, D. (1995): "Aplicación de las técnicas mineralógicas y petrológicas a la Arqueometría. Estudio de los materiales del dolmen de Alberite (Villamartín, Cádiz)" Zephyrus, XLVIII: 29-142.

EASTAUGH, N.; WALSH, V.; CHAPLIN, T. Y SIDDALL, R. (2005): The Pigment Compendium. Elsevier, Amsterdam.

FÁBREGAS VALCARCE, R. y PÉREZ LOSADA, F. y FERNÁNDEZ IBÁNEZ, C. (eds.) (1995): Arqueología da Morte na Península Ibérica desde as Orixes ata o Medievo. Excmo. Concello Xinxo de Limia.

FABIÁN GARCÍA, J. F. (1992): "El enterramiento campaniforme del Túmulo 1 de Aldeagordillo (Ávila)". B.S.S.A., LVII: 97-132.

FABIÁN, J. F. (2006): El IV y el II milenio AC en el Valle Amblés (Ávila). Monografías Arqueología en Castilla y León 5. Junta de Castilla y León.

FLORES, R. (2011): "El yacimiento de Humanejos (Parla, Madrid)". En Blasco et al. (eds.): 9-16.

GALINDO SANJOSÉ, L. SÁNCHEZ SÁNCHEZ, V.M. y LORENTE DE PEÑA, M. (2009): "Soto de Henares: aproximación a un poblado de recinotos". Actas de las Cuarta Jornadas de Patrimonio Arqueológico de la Comunidad de Madrid. Dirección General de Patrimonio Histórico y Museo Arqueológico Regional, Madrid: 263-271.

GARCÍA BORJA P.; DOMINGO SANZ, I. y ROLDÁN GARCÍA, C. (2006): nuevos datos sobre el uso de materia colorante durante el Neolítico Antiguo en las comarcas centrales valencianas. Saguntum (P.L.A.V.), 38: $49-60$.

GARRIDO, R. (1999): El campaniforme en la meseta Central: Análisis de su contexto social, económico y ritual. Tesis doctoral. Universidad Complutense de Madrid.

GARRIDO, R. (2000): El campaniforme en la meseta Central de la península Ibérica. (c 2500-2000 A.C.), BAR International series 892 . Oxford

GÓMEZ, J. 1., BLASCO, C., TRANCHO, G., GRUESO, I., RÍOS; P. y MARTÍNEZ ÁVILA, M. (2011): "Los protagonistas". En Blasco et al. (eds.), 101-132.

GUERRA, E. (2006): "Sobre la función y el significado de la ceráminca campaniforme la luz de los análisis de contenidos. Trabajos de Prehistoria, 63, (1): 69-84

GUERRA, E. y DELIBES, G. (2004): “Contexto y posible significado de un cuenco Ciempozuelos con decoración simbólica de ciervos hallado en Almenara de Adaja (Valladolid)" Zona Arqueológica 4, 4 (Miscelánea en Homenaje a Emiliano Aguirre. Arqueología), 116-125. 
HARRISON, R. (1977): The Bell Beaker Cultures of Spain and Portugal. American School of Prehistoric Research Bulletin 36. CambridgeMassachussets.

HARRISON, R. y GILMAN, A., (1977): "Trade in the second and third millennia b.C. between the Magreb and Iberia”. En: Ancient Europe and the Mediterranean. Studies in honour of Hugo Wencker, Warminster, 91-104

HARTMANN, A. (1982): Prähistoriche Goldfunde aus Europa II. Spektralanalytischische Unterschungen und deren Auswertung, S.A.M. 5 Gebr. Mann, Berlín.

HERNANDO, A. (1983): “La orfebrería durante el Calcolítico y el Bronce Antiguo en la Peninsula Ibérica". Trabajos de Prehistoria 40, 83-138.

HERNANDO, A. (1989): "Inicios de la orfebrería en la Península Ibérica" El oro en la España prerromana. Revista de Arqueología: 32-45.

HUNT, M. y HURTADO, V. (2010): Pigmentos de sulfuro de mercurio -cinabrio- en contextos funerarios de época calcolítica en el sur de la península ibérica: investigaciones sobre el uso, depósitos minerales explotados y redes de distribución a través de la caracterización composicional e isotópica. VIII Congreso Internacional de Arqueometría. Sesión pigmentos: 123-132.

LIESAU, C. (2011): Los restos de mamíferos del ámbito doméstico y funerario. En Blasco et al., (eds.): 171-189.

LIESAU VON LETTOW-VORBECK, C. y PASTOR ABASCAL， I. (2003): "The Ciempozuelos Necropolis Skull: A Case of Double Trepanation? International Journal of Osteoarchaeology, 13:213-221.

LIESAU, C., BLASCO, C., RÍOS, P., VEGA , J., MENDUIÑA, R., BLANCO, J.F., BAENA, J., HERRERA, T., PETRI, A. y GÓMEZ, J.L (2008): "Un espacio compartido por vivos y muertos: el poblado calcolítico de fosos de Camino de las Yeseras". Commplutum, vol 19-I, 97-120.

LIESAU, C. y MORENO E. (2011): Marfiles campaniformes de El Camino de las Yeseras (San Fernando de Henares, Madrid), En: Th. X. Schuhmacher- D. Marzoli- J. A. López Padilla (Eds.): Marfil y Elefantes en la Península Ibérica y el Mediterráneo, Coloquio Internacional. Museo Arqueológico de Alicante. Iberia Archaeologica, 16: 87-98.

LIESAU, C. y SCHUHMACHER, T.X. (2011): Un taller de marfil en el yacimiento argárico de Fuente Álamo (Cuevas de Almanzora, Almería).
En: Th. X. Schuhmacher- D. Marzoli- J. A. López Padilla (Eds.): Marfil y Elefantes en la Península Ibérica y el Mediterráneo, Coloquio Internacional. Museo Arqueológico de Alicante, Iberia Archaeologica , 16: 125-141.

LIESAU, C.; BANERJEE, A. y SCHWARZ, J.O. (2011): Camino de las Yeseras' ivory collection: advances in analysis technology used in identifying raw material. En Blasco et al. (eds): 381386.

LOMBA, J., LÓPEZ M., RAMOS F., y AVILÉS FERNÁNDEZ A. (2009): "El enterramiento múltiple, calcolítico, de Camino del Molino (Caravaca, Murcia). Metodología y primeros resultados de un yacimiento excepcional", Trabajos de Prehistoria, 66, vol. 2, 143-159.

LÓPEZ SAEZ, J.A. (2011): "Análisis palinológico". En Blasco et al. (eds.), 251-260.

LÓPEZ PADILLA, J.A. (2006a):'Distribución territorial y consumo de botones de perforación en "V" en el ámbito argárico". Trabajos de Prehistoria, 63,2: 93-116.

LÓPEZ PADILLA, J.A. (2006b): "Marfil, oro, botones y adornos en el área oriental del país de El Argar". MARQ, Arqueología Museos, 1: 25-48.

MÁRQUEZ, J. (2001) "De los campos de silos a los agujeros negros: sobre pozos, depósitos y zanjas en la Prehistoria Reciente del sur peninsular", Spal, $\mathrm{n}^{\circ}$ 10. Universidad de Sevilla, 207-220.

MÁRQUEZ, J. (2006): "Sobre los depósitos estructurados de animales en yacimientos de fosos del Sur de la Península Ibérica". Animais na Pré-hisória e Arqueología da Península Ibérica. Actas do IV Congresso de Arqueología Peninsular, 15-25. Braga.

MÁRQUEZ, J.E. y JIMÉNEZ, V. (2010): recintos de fosos. Genealogía y significado de una tradición en la Prehistoria del suroeste de la Península Ibérica (IV-III milenios A.C.). Universidad de Málaga.

MONTERO, I. y ROVIRA, S. (1991): "El oro y sus aleaciones en la orfebrería prerromana". Archivo Español de Arqueología 64: 7-21.

PEÑA, L., RUIZ, M. y SÁBATO, D (2011): “Los macrorrestos vegetales". En: Blasco et al. (eds.), 261-276.

PEREA, A. (1989): “Tecnología y métodos de estudio". En: El oro en la España prerromana. Revista de Arqueología: 24-31.

PEREA, A. (1991): Orfebrería prerromana. Arqueología del oro. Madrid. 
PÉREZ ARRONDO, C. y LÓPEZ DE CALLE, C(1986): "Aportaciones al estudio de las culturas eneolíticas en el Valle del Ebro. I: Elementos de adorno". Instituto de Estudios riojanos. Logroño.

RIAÑO, J.F, RADA, J. y CATALINA J. (1894): Hallazgo prehistórico en Ciempozuelos. Boletín de la Real Academia de la Historia , XXV: 436450.

RÍOS, P. y LIESAU, C., (2011): "Elementos de adorno simbólicos y colorantes en contextos funerarios y singulares”. En Blasco et al. (eds.), 357-370.

RÍOS, P., (2011): Territorio y sociedad en la Región de Madrid durante el III milenio a.C. El referente del yacimiento de Camino de las Yeseras. Patrimonio Arqueológico de Madrid, 7. Universidad Autónoma de Madrid.

RODRÍGUEZ DE LA ESPERANZA, M ${ }^{\mathrm{a}}$ J. (2004): "La primera industria del oro en el Valle del Ebro”. Anejos al Archivo Español de Arqueología XXXII: 189-195.

ROJO GUERRA, M.; GARRIDO PENA, R.; GARCÍA-MARTÍNEZ-DE-LAGRÁN, I. TRESERRAS, J.J. y MATAMALA, J.C. (2006): “Beer and Bell Beakers: Drinking Rituals in Copper Age Inner Iberia". Proceedings of the Prehistoric Society, 72:243-265.

ROVIRA, S. y SANZ; M. (1984): “Análisis de Laboratorio de algunos materiales de la cueva de Juan Barbero". Apéndice 4 en MARTINEZ NAVARRETE, M $\mathrm{M}^{\mathrm{a}}$ I.: "El comienzo de la metalurgia en la provincia de Madrid: La Cueva y cerro de Juan Barbero (Tielmes Madrid)". Trabajos de Prehistoria 41, 17-128.

SALANOVA, L. y TCHÉRÉMISSINOFF, Y. (dirs.) (2011): Les sépultures individuelles campaniformes en France. Gallia Préhistoire XLI Supplément. CNRS éditions, París.
SAMPEDRO, C. y LIESAU, C. (1998): El yacimiento campaniforme de la Cuesta de la Reina (Ciempozuelos). Los restos antropológicos. En: Blasco et al. (eds.): 334-355.

SÁNCHEZ PALENCIA. F. J. y PÉREZ, L. C. 1989: "Los yacimientos auríferos de la Península Ibérica. Posibilidades de explotación en la Antigüedad". En: El oro en la España prerromana. Revista de Arqueología: 16-23.

SIRET, H. y SIRET, L. (1890): La Edad de los Metales en el Sudeste de España. Barcelona.

SCHUHMACHER, T.X. y CARDOSO, J.L (2007): Ivory objects from the chalcolithic fortification of Leceia (Oeiras). Estudos Arqueológicos de Oeiras, 15 Oeiras, Camara Municipal, 2007, 95118.

SCHUHMACHER, T.X.; BANERJEE, A. y CARDOSO, J.L (2009): Sourcing African ivory in Chalolithic Portugal. Antiquity, 83, no 322: 983997.

TCHÉRÉMISSINOF, ESCALON, G. y DONAT, R. (2011): "Le coffre lithique campaniforme ou épicampaniforme du site Georges Besse II-5. Nîmes (Gard)". En Salanova y Tchérémissinoff (dirs.), 165-176.

USCATESCU, A. (1992): Los botones perforados en "V" en la Península Ibérica y Baleares durante la Edad de los Metales. Temas de Arqueología, 2, Foro Arqueología, Proyectos y Publicaciones, Madrid, 1992.

VEGA, J.; BLASCO, C.; LIESAU, C.; RÍOS, P.; BLANCO, J.F.; MENDUIÑA, R.; ALIAGA, R.; MORENO, E.; HERRERA, T.; PETRI, A. y GÓMEZ, J.L.: "La singular dualidad de enterramientos en el poblado de silos calcoítico de Camino de las Yeseras (San Fernando, Madrid). Munibe, suplemento, 32: 648-662. 